\title{
Localization Using Joint AOD and RSS Method in Massive MIMO System
}

\author{
Wan Amirul MAHYIDDIN ${ }^{1}$, Ahmad Loqman AHMAD MAZUKI ${ }^{1,2}$, Kaharudin DIMYATI ${ }^{1}$, \\ Mohamadariff OTHMAN ${ }^{1}$, Norrima MOKHTAR ${ }^{1}$, Hamzah AROF $^{1}$ \\ ${ }^{1}$ Dept. of Electrical Engineering, University of Malaya, 50600 Kuala Lumpur, Malaysia \\ ${ }^{2}$ Dept. of Electrical \& Electronics Engineering, Universiti Pertahanan Nasional Malaysia, 57000 Kuala Lumpur, Malaysia \\ wanamirul@um.edu.my
}

Submitted May 22, 2019 / Accepted October 10, 2019

\begin{abstract}
In this research, we propose to estimate location of mobile users by using joint angle of departure (AOD) from base station (BS) and received signal strength (RSS) at user equipment (UE) method. The location estimation (LE) is performed at each UE by using massive multipleinput multiple-output (MIMO) system at the BS, which transmits specially design MIMO-orthogonal frequency division multiplexing (MIMO-OFDM) beamforming signals. Since the estimation is done independently at each $U E$, the number of UEs in the area does not affect the performance and calculation complexity of the LE. To improve the practicality of the proposed method, we also design the beamforming signals with reduced peak to average power ratio (PAPR) by using random subcarrier-beamforming angle allocation method. The results show that the proposed method generally has lower estimation error than that of the AOD-only and the RSS-only LE methods across various UEs' locations. The proposed beamforming signal method can also significantly reduce PAPR of the transmitted signal.
\end{abstract}

\section{Keywords}

Localization, massive MIMO, mobile positioning, OFDM

\section{Introduction}

Mobile positioning system has becoming a crucial part of our life partly due to the impressive growth of smartphones usage within the past decade. With the recent increase in consumers' interests for Internet of Things (IoT), quadcopters (drones) and autonomous vehicles, we expect that the positioning technology will continue to increase its presence in our daily life. Despite its importance, the current positioning system still does not perform very well when it comes to detecting a location of a device within an urban area that comprises of dense highrise buildings or indoor settings. This is due to its dependence on Global Positioning System (GPS) which uses satellite signals for location detection [1]. These signals will be severely attenuated when they reach to an area that is surrounded with dense obstacles or indoor location [2], [3].

Recently, there has been an increase of interest to improve positioning system by using the next generation wireless communication technology, namely with massive MIMO systems [4-8]. The idea is to use access points (APs) or BSs that can act as both communication and positioning systems. Since the wireless technology is also moving towards high density APs systems such as small cells [9], distributed antennas [10] and relay networks [11], we expect that more line of sight (LOS) signals exchange can be achieved between APs and mobile users as compared to GPS signals. For GPS, the location of a UE is estimated by using time of arrival (TOA) of signals from the GPS satellites. Since electromagnetic signal travels at the speed of light, even a small TOA measurement error can result to significant LE error. This means that expensive hardware with atomic clock precision must be used for LE using GPS signals [1]. As a result, similar GPS technology may not be economically viable for large scale implementations of LE using cellular network.

In addition of TOA, LE can also be achieved by using RSS or power of arrival (POA) [12], [13]. By using the measured power, we can estimate the distance between transmitter and receiver with the assistance of path-loss model. In massive MIMO system, signal beamforming can be used to increase the desired signal at the target device and reduce the interference at the non-target area [14]. However, various issues such as signal attenuation from obstacles and multipath propagations may cause inaccurate LE. Another important advantage of massive MIMO system is it has high angle estimation accuracy. This property had been used in [4] where multiple BSs cooperatively perform signal processing to estimate the location of a UE. However, in [4], obtaining LE for large number of UEs will require more time-frequency resources and higher calculation complexity. In [5], [6], angle-based method has been proposed to perform LE with only one BS. However, this method requires large number of antennas at both transmitter and receiver. Massive MIMO with fingerprinting method, which uses spatial signature to estimate the UEs' locations, has also been proposed [7], [8]. The challenge of 
the fingerprinting method is it requires data training and complex recognition algorithm such as neural network to estimate the location [8].

The main contribution of this paper is to perform LE that uses joint AOD and RSS estimation method at the UEs by using an adaptive algorithm. Each UE can perform the LE by using the proposed MIMO-OFDM signal received from the BS. Unlike the methods that do the LE at the BSs or the APs [4], [7], [8], our proposed LE method is done independently at each UE, which means that higher number of UEs does neither reduce the LE's accuracy nor increase its calculation complexity at the BSs. LE method that uses the angle between BSs and UEs has also being investigated [4]. However, our results show that such LE method will perform poorly when there are only 2 reliable BSs and the $\mathrm{UE}$ is located near the straight line that connects the $2 \mathrm{BSs}$. Meanwhile, in the case of an RSS-based LE [12], [13], the method may perform poorly due to signal attenuation, noise, interference and multipath channels [3]. Hence, in this paper, we take advantage of both of the LE methods by designing an algorithm that can detect the reliability of angle-based LE and switch to power-based LE under certain conditions. Our additional contribution in this research is to design the OFDM beamforming signal with reduced PAPR in order to improve the practicality of the proposed method.

Notation: $|\cdot|, \operatorname{Im}(\cdot), \operatorname{Re}(\cdot)$ and $\arg (\cdot)$ are element-wise absolute value, imaginary value, real value and angle of complex number, respectively. Boldface variables represent vector, $\mathcal{C N}(a, b)$ is the complex Gaussian distribution with mean $a$ and variance $b,\{\cdot\}_{x}$ is the $x$-th element of the vector or the set, $(\cdot)^{\mathrm{H}}$ is Hermitian transpose and '。' is Hadamard product.

\section{System Model}

\subsection{Channel Response}

We use $M$ antennas at each BS and one antenna at each UE. We assume that the transmissions contain both LOS and NLOS signals as shown in Fig. 1.

In Fig. 1, the signals can arrive at the UE from multiple number of AODs from BS due to the presence of scatterers and reflectors in the surrounding environment, which create NLOS signals. Note that when signals are transmitted

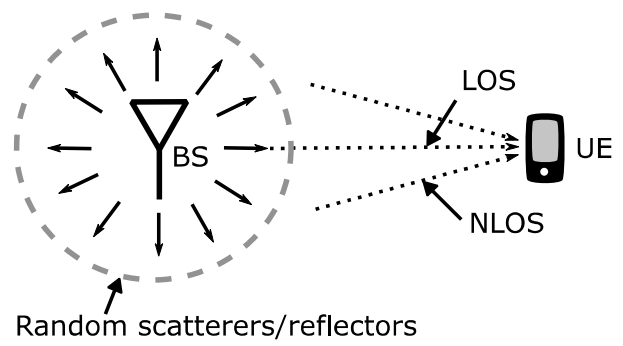

$\overrightarrow{\text { Transmitted }}$ signal Arrived signal

Fig. 1. Signal transmitted from BS to UE.

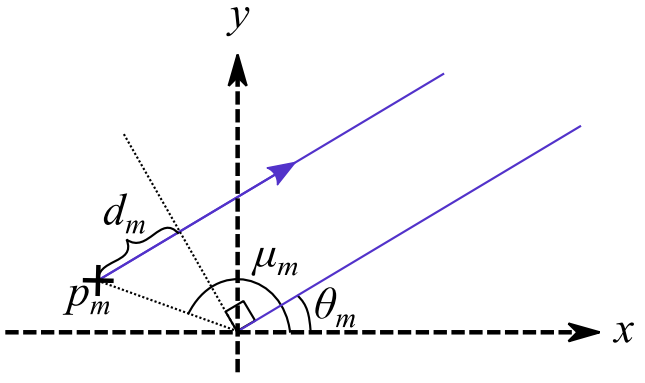

Fig. 2. Signal transmitted from a single antenna $m$ at the BS.

between BS and UE, there is only one AOD for LOS (straight line between BS and UE) while there can be multiple AODs for NLOS. Each AOD has different channel response, depending on the position of the antennas. In this paper, we use complex number to represent the $2 \mathrm{D}$ coordinate system. The location of an antenna with index number $m$ is defined as

$$
p_{m}=p_{m, x}+\mathrm{j} p_{m, y}
$$

where $p_{m, x}$ and $p_{m, y}$ are the $x$ and $y$ coordinates of antenna $m$, respectively, with the center of mass of the antenna system as the origin. The coordinates of all antennas for a certain BS are grouped into a vector form as

$$
\mathbf{p}=\left[p_{1}, p_{2}, \ldots, p_{M}\right] .
$$

The coordinates in (2) are dependent on the number of antennas, spacing between antennas and the arrangement of antennas such as circular or linear. In this research, we use circular antennas arrangement.

Figure 2 illustrates the electromagnetic signal transmission from one antenna $m$, where $p_{m}$ is the local coordinate of the antenna, $d_{m}$ is the signal travelling distance difference between the antenna and the origin, $\theta_{m}$ is the signal AOD and $\mu_{m}$ is the antenna's angle with respect of the $\mathrm{x}$-axis of the origin. In order to build the channel response for each antenna, we need to consider the phase differences between different antennas. The channel response of the same signal's path for different antennas may have different phases due to the difference in the signal's travelling distance. The difference of travelling distance with respect of the origin can be calculated as $d_{m}=\left|p_{m}\right| \sin \left(\mu_{m}-\pi / 2-\theta_{m}\right)$. Hence, the phase difference of antenna $m$ with respect of the origin is $\phi_{m}=2 \pi d_{m} f_{\mathrm{C}} / c$, where $f_{\mathrm{C}}$ is the carrier's frequency and $c$ is the speed of light. If we extend this concept to the multiple antennas system, the $1 \times M$ vector of signal phase difference with respect of the origin can be expressed as

$$
\begin{aligned}
\phi(\mathbf{p}, \theta) & =2 \pi|\mathbf{p}| \circ \sin \left(\arg (\mathbf{p})-\frac{\pi}{2}-\theta\right) \frac{f_{\mathrm{C}}}{c} \\
& =-2 \pi|\mathbf{p}| \circ \cos (\arg (\mathbf{p})-\theta) \frac{f_{\mathrm{C}}}{c}
\end{aligned}
$$

where $\mathbf{p}$ is defined in (2). Note that all the mathematical operations that involve vectors in (3) are element-wise operations. 
In the case of multipath channel propagations, the multiple copies of same signal may arrive at the same antenna but with different delays. To include the multipath delay effect, we can rewrite (3) as follows

$$
\phi(\mathbf{p}, \theta, \tau)=2 \pi f_{\mathrm{C}}\left(-|\mathbf{p}| \circ \cos (\arg (\mathbf{p})-\theta) \frac{1}{c}+\tau\right)
$$

where $\tau$ is the time delay of a certain signal's path with respect of the first arrived signal. We set the first arrived signal as the reference point where the delay is zero, $\tau=0$. The first arrived signal is assumed as LOS signal since it has the shortest path and the shortest travelling time. From (4), we can write the small-scale fading of the LOS channel response as

$$
\mathbf{h}(\tau)=\exp [\mathrm{j} \phi(\mathbf{p}, \theta, \tau)] \text { for } \tau=0 .
$$

In the case of NLOS channel, the signal can depart from the BS's antennas from multiple angles. So the total smallscale fading of NLOS channel response at delay $\tau$ can be written as

$$
\mathbf{h}(\tau)=\frac{1}{\sqrt{L}} \sum_{l=1}^{L} a_{\tau, l} \exp \left[\mathrm{j} \phi\left(\mathbf{p}, \theta_{l}, \tau\right)\right] \text { for } \tau>0
$$

where $L$ is the total number of different signal paths at the time delay $\tau$, and $a_{\tau, l}$ is the complex valued amplitude of the $l$-th signal at delay $\tau . a_{\tau, l}$ is the channel amplitude based on exponential decay model and value of $\tau$ for each path is generated based on random Poisson distribution [16]. The parameter values for the channel model will be further discussed in the numerical results section.

Using the tapped-delay channel response equations in (5) and (6), the summation of signals that arrive at the UE at discrete time $n$ can be expressed as

$$
y_{n}=\sum_{\tau=0}^{T} \sqrt{P_{\mathrm{B}} \beta} \mathbf{h}_{\tau} \mathbf{s}_{\tau-n}+w
$$

where $P_{\mathrm{B}}$ is the average transmit power normalized to the noise power at the $\mathrm{BS}$ and $w$ is complex Gaussian noise signal with $\mathcal{C N}(0,1)$ distribution. $\beta$ is the large-scale fading that is generated according to path-loss model in the results section. $\mathbf{s}_{\tau-n}$ is the MIMO-OFDM signal in time domain which will be discussed in the next section.

\subsection{Transmitted Signal at BS}

To perform LE using AODs, angle estimation must be done at the UE that has at least 2 BSs with LOS signal transmission. The basic idea of the proposed AOD estimation method is that during the transmission, the signal is beamformed at every possible discrete angle with equal angle spacing. This is done by using unique beamforming vector for every OFDM subcarrier signal. We define the beamforming angle as

$$
\boldsymbol{\theta}=\left[\theta_{1}, \theta_{2}, \ldots \theta_{a}, \ldots \theta_{N}\right]
$$

where $\theta_{a}=2 \pi a / N$ and $N$ is the total number of discrete angles. Note that based on our initial simulation, if the beamforming angle of the OFDM subcarrier signal increases linearly with respect to subcarrier number, this will result to a very high PAPR. Specifically, in our simulation settings, linear subcarrier-beamforming angle allocation has produced $16 \mathrm{~dB}$ PAPR. Such high PAPR means that the beamforming method will not perform very well for non-linear transceiver. To reduce the PAPR, we propose to use random mapping between beamforming angle and the subcarrier number. To achieve this, we shuffle the elements in vector $\boldsymbol{\theta}$ randomly as follows

$$
\boldsymbol{\theta}^{\prime}=\operatorname{shuffle}(\boldsymbol{\theta}) \text {. }
$$

Even though the shuffle function (9) relocates the elements of the vector at random positions, we can recover back the original positions by using reverse shuffle function as follows

$$
\boldsymbol{\theta}=\text { reverse_shuffle }(\text { shuffle }(\boldsymbol{\theta})) \text {. }
$$

Once the elements in $\boldsymbol{\theta}$ are shuffled, we then form the beamforming vector at angle $\{\boldsymbol{\theta}\}_{k}$ as

$$
\mathbf{b}_{k}=\left(\exp \left[\mathrm{j} \phi\left(\mathbf{p},\left\{\boldsymbol{\theta}^{\prime}\right\}_{k}, \tau\right)\right]\right)^{\mathrm{H}} .
$$

$\mathbf{b}_{k}$ is a $1 \times M$ vector because there are $M$ number of antennas at the BS and each antenna transmits multiple number of subcarriers (different $k$ ) at the same time.

To perform OFDM transmission through a wireless channel, the signal must be converted from subcarrier domain to time domain. The transmitted OFDM signal at discrete time $n$ from $M$ number of BS's antennas can be expressed as [15]

$$
\mathbf{s}_{n}=\frac{1}{N} \sum_{k=0}^{N-1} \mathbf{X}_{k} \exp \left[\mathrm{j} \frac{2 \pi k n}{N}\right]
$$

where $\mathbf{X}_{k}$ is $M \times 1$ signal vector at subcarrier $k$ for $M$ number of antennas. Since we aim to set the subcarrier $k$ to beamform the signal at angle $\left\{\boldsymbol{\theta}^{\prime}\right\}_{k}$, this means that $\mathbf{X}_{k}=\mathbf{b}_{k}$. The generated value of $\mathbf{s}_{n}$ from (12) will be used in (7) to obtain the received signal at the UE.

\section{Location Estimation}

In this section, we discuss the two LE methods, which are AOD and RSS methods. The fundamental concept of our LE method is to use the MIMO-OFDM beamforming signal from the BS that is specifically designed to enable the AOD estimation of the signal at the UE. We will also discuss the joint AOD and RSS method, where the AOD method can be switched to the RSS method under certain condition.

\subsection{LE using Angle of Departure (AOD)}

Once the signal in (12) is transmitted from the BS and arrived at the UE through the channel model in (7), then the OFDM signal is converted from time domain to subcarrier 
domain by applying DFT [15], as follows

$$
X_{f}=\sum_{n=0}^{N-1} y_{n} \exp \left[-\mathrm{j} \frac{2 \pi f n}{N}\right] \text {. }
$$

We define $\mathbf{X}=\left[X_{1}, X_{2}, \ldots, X_{f}, \ldots, X_{F}\right]$ as the signal vector in subcarrier domain after DFT. Note that $\mathbf{X}$ is the signal with shuffled beamforming angle to subcarrier mapping based on (9). To arrange the signal in the correct order of angle, we apply reverse shuffle (10), which aims to reverse the random subcarrier-beamforming angle mapping, as follows

$$
\mathbf{X}^{\prime}=\text { reverse_shuffle }(\mathbf{X}) \text {. }
$$

As we discussed previously, we depend on LOS signal to estimate the angle along with the assumption that LOS signal's path has the largest RSS. Therefore, to find the AOD of the LOS signal, we find the element number in $\mathbf{X}^{\prime}$ that has the highest value, as follows

$$
a=\underset{a}{\operatorname{argmax}}\left(\left\{\mathbf{X}^{\prime}\right\}_{a}\right)
$$

From (15), the estimate of AOD can be expressed as

$$
\hat{\theta}=\{\boldsymbol{\theta}\}_{a}
$$

where $\boldsymbol{\theta}$ is defined at (8).

Note that (16) is the angle estimation from one BS. For location estimation, signals from two BSs should be used to provide UE position determination at the intersection point of two angle estimations from the two BSs. Let say that $\hat{q}_{u}$ is defined as the estimated coordinate of the UE in complex number. Using a simple trigonometric calculation, the AOD can be expressed as $\tan (\hat{\theta})=\left[\operatorname{Im}\left(\hat{q}_{u}\right)-\operatorname{Im}\left(q_{b}\right)\right] /\left[\operatorname{Re}\left(\hat{q}_{u}\right)-\operatorname{Re}\left(q_{b}\right)\right]$, where $q_{b}$ is coordinate of BS $b$. Using the estimated AODs from BS $b_{1}$ and $b_{2}\left(\hat{\theta}_{b_{1}}\right.$ and $\left.\hat{\theta}_{b_{2}}\right)$ along with the locations information of the BSs, the estimated UE location, $\hat{q}_{u}$ can be solved as

$$
\begin{aligned}
& {\left[\begin{array}{l}
\operatorname{Im}\left(\hat{q}_{u}\right) \\
\operatorname{Re}\left(\hat{q}_{u}\right)
\end{array}\right]=} \\
& {\left[\begin{array}{ll}
1, & -\tan \left(\hat{\theta}_{b_{1}}\right) \\
1, & -\tan \left(\hat{\theta}_{b_{2}}\right)
\end{array}\right]^{-1} \times\left[\begin{array}{l}
\left(\operatorname{Im}\left(q_{b_{1}}\right)-\tan \left(\hat{\theta}_{b_{1}}\right) \operatorname{Re}\left(q_{b_{1}}\right)\right) \\
\left(\operatorname{Im}\left(q_{b_{2}}\right)-\tan \left(\hat{\theta}_{b_{2}}\right) \operatorname{Re}\left(q_{b_{2}}\right)\right)
\end{array}\right]}
\end{aligned}
$$

where $q_{b_{1}}$ and $q_{b_{2}}$ are the coordinates for BS $b_{1}$ and BS $b_{2}$ in complex number, respectively. In order to simplify this paper, we define the estimated position as

$$
\hat{q}_{u}=\operatorname{LE}\left(\hat{\theta}_{b_{1}}, \hat{\theta}_{b_{2}}, q_{b_{1}}, q_{b_{2}}\right)
$$

where $\mathrm{LE}(\cdot)$ is the function that performs the calculation in (17).

\subsection{LE using Received Signal Strength (RSS)}

To estimate the UE position from RSS, we use OFDM signal from one BS. The UE will receive the OFDM signal and obtain results in (14) to calculate the large-scale fading of LOS. As mentioned previously, signal that has LOS beamforming angle has higher power than that of NLOS. Hence, to estimate the distance, we must estimate the large-scale fading of LOS signal, $\hat{\beta}$, as follow

$$
\hat{\beta}=\max \left(\mathbf{X}^{\prime}\right) /\left(M P_{\mathrm{B}}\right) .
$$

$M P_{\mathrm{B}}$ in (19) is to normalize the received power from the multiple antennas transmission. In this paper, we exclude the antenna gain effect. However, such gain can also be measured and normalized at the receiver. Using free-space path-loss model for LOS signal [15], we can estimate the distance as

$$
\hat{d}=\lambda /\left(4 \pi \hat{\beta}^{1 / 2}\right)
$$

Once we obtain $\hat{d}$, the location can be calculated as

$$
\left[\begin{array}{l}
\operatorname{Im}\left(\hat{q}_{u}\right) \\
\operatorname{Re}\left(\hat{q}_{u}\right)
\end{array}\right]=\left[\begin{array}{l}
\operatorname{Im}\left(q_{b_{1}}\right)+\hat{d} \sin (\hat{\theta}) \\
\operatorname{Re}\left(q_{b_{1}}\right)+\hat{d} \cos (\hat{\theta})
\end{array}\right]
$$

where $\hat{\theta}$ is the estimated angle between the UE and the BS from (16). Technically, both AOD and RSS are used to estimate the location in (21). However, to avoid confusion with the proposed joint AOD and RSS method (discussed in the next section), we define LE using (21) as RSS-only method.

\subsection{LE using Joint AOD and RSS}

Even though LE can be done by using AODs, this estimation method may result to a high error if the UE is located at a certain position. Specifically, if the UE is located on or near the direct line between the two BSs, location estimation using AOD will be unreliable. This is because at this location, even a small variation of angle can cause a large change in the estimated position. Therefore, we propose an algorithm to determine the reliability of the estimation using (18), as described in Algorithm 1.

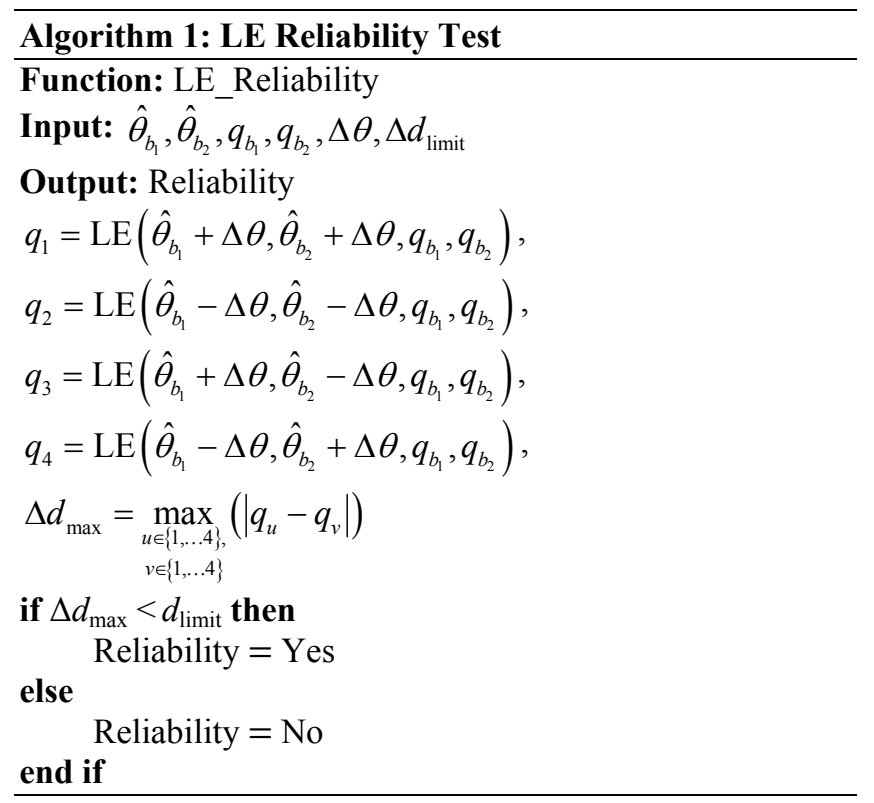


$\Delta d_{\max }$ is the maximum distance deviation as a result of the angle variation of $\pm \Delta \theta$ from both BSs. $\Delta \theta$ is set to half of angle spacing between the beamforming angle in (8). $\Delta d_{\text {limit }}$ is the distance deviation limit to ensure the reliability. If $\Delta d_{\max }$ is less than $\Delta d_{\text {limit }}$, then the location estimation is considered as reliable. In this research, we set $\Delta d_{\text {limit }}$ to 1 meter. Using this algorithm, the value of $\Delta d_{\max }$ will change dynamically depending on the estimated location of the UE. For example, if the estimated location is near the direct line between the BSs, the $\Delta d_{\max }$ value becomes large, and vice versa. This algorithm also means that if there are multiple BSs with LOS signals to the UE, the UE will accept the reliable LE only. However, if there is no reliable LE using the AOD method, then the LE will be done using RSS method. The complete procedure of the proposed LE using joint AOD and RSS is described in Algorithm 2.

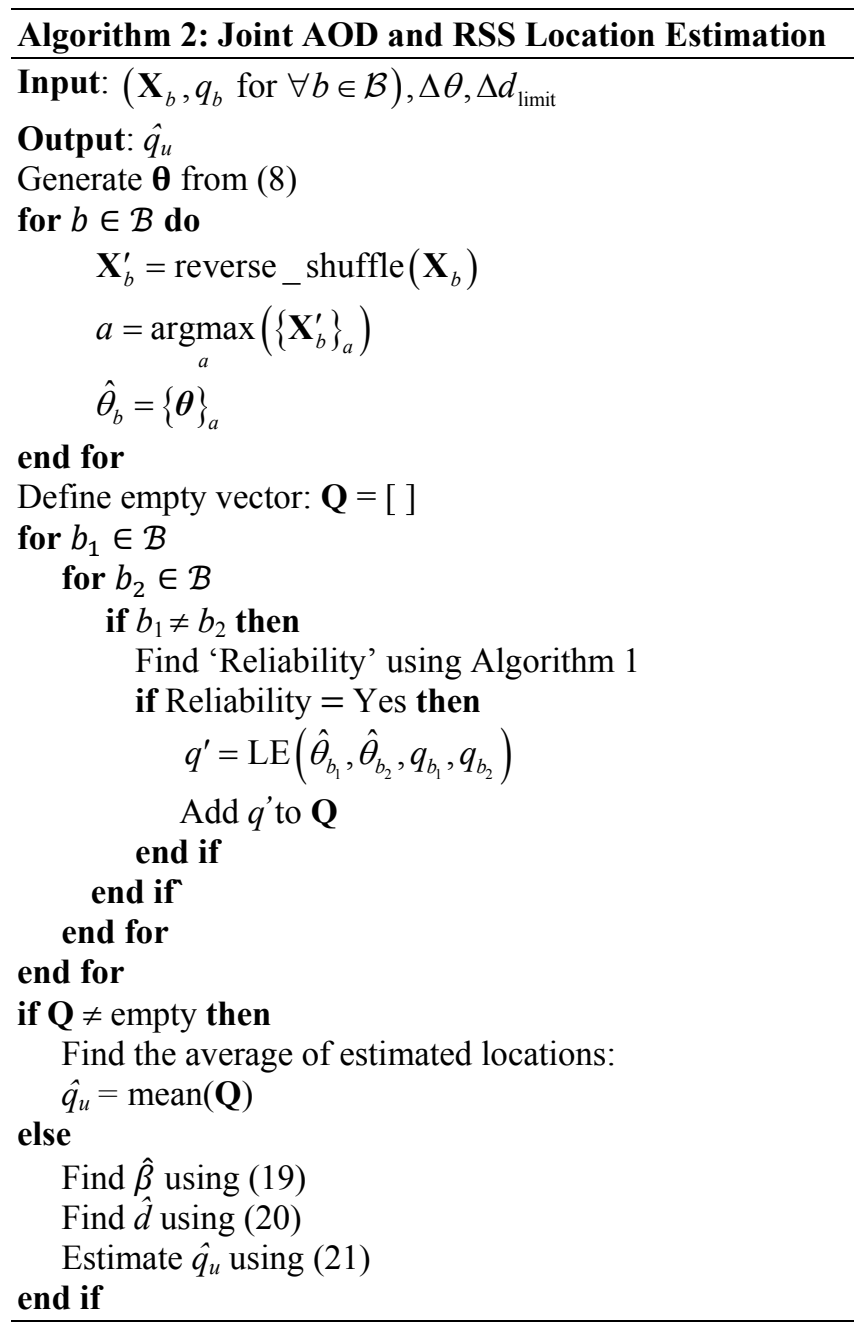

$\mathbf{X}_{b}$ is the received OFDM signal from BS $b$ after applying $\operatorname{DFT}(13), q_{b}$ is the location of $\mathrm{BS} b, \mathcal{B}$ is defined as a set of $\mathrm{BS}$ index numbers in the coverage area.

\section{Results}

For each BS, we use massive MIMO system with 100 antennas that are arranged in circular shape with a half wavelength separation between adjacent antennas. The transmit power of each BS is $24 \mathrm{dBm}$. For large-scale fading, $\beta$, we use ITU indoor path-loss model, Loss $=$ $20 \log _{10} f_{\mathrm{C}}+N \log _{10}(d)+P_{\mathrm{f}}(n)-28 \mathrm{~dB}$, where $f_{\mathrm{C}}$ is the carrier frequency in megahertz, $N$ is the distance power loss coefficient, $d$ is the distance between BS and UE and $P_{\mathrm{f}}(n)$ is the floor loss penetration factor (set to be zero for the same floor) [17]. For $N$, we set it to be 20 for LOS (free space path-loss) and 22 for NLOS (indoor commercial area). The bandwidth is $20 \mathrm{MHz}$, and carrier frequency is $f_{\mathrm{C}}=2 \mathrm{GHz}$. The noise power in the bandwidth $B$ can be calculated using Boltzmann formula, which is $P_{\mathrm{N}}=k_{\mathrm{b}} T_{0} B N_{\mathrm{F}}$, where $k_{\mathrm{b}}=1.381 \times 10^{23} \mathrm{~J} / \mathrm{K}, \quad T_{0}=200 \mathrm{~K}$, $N_{\mathrm{F}(\mathrm{dB})}=9 \mathrm{~dB}$ [18]. For the simulation environment, the multipath channels are generated according to the statistical indoor channel model from [16]. We use 10 clusters of multipath channels and each cluster contains 10 rays of sub-path channels. The AODs of each NLOS signal cluster are generated at random angles. We set the cluster arrival rate to $1 / 17 \mathrm{~ns}$, the ray arrival rate to $1 / 5 \mathrm{~ns}$, the cluster decay rate to $34 \mathrm{~ns}$ and ray decay rate to $29 \mathrm{~ns}$ [4]. We set the number of discrete beamforming angles in (8) to $N=2048$. We also add random bias to $\boldsymbol{\theta}$ in (8) in order to obtain smoother statistical curves results. The spacing between BSs is set to 80 meters, which corresponds to indoor setting [4]. The locations of BSs and UEs are shown in Fig. 3.

In Fig. 3, the UEs are arranged between $-20 \mathrm{~m}$ and $20 \mathrm{~m}$ across the $\mathrm{x}$-axis. There is $2 \mathrm{~m}$ spacing between adjacent UEs. However, we reduce the spacing to $0.5 \mathrm{~m}$ within $-5 \mathrm{~m}$ and $5 \mathrm{~m}$ across the $\mathrm{x}$-axis due to higher RMSE curve's gradient within this region. The UEs' positions are also varied across the $y$-axis by positioning the UEs at the center $(y=0)$ and at the edge $(y=-30)$ of the y-axis. To test the performance of the proposed method, we measure the LE accuracy performance by using root mean square error (RMSE) of the estimation. Lower RMSE means better LE accuracy. For each UE location, we simulate 5000 channel realizations. The RMSE of LE results for UEs at the center and the edge of y-axis are given in Fig. 4 and 5, respectively.

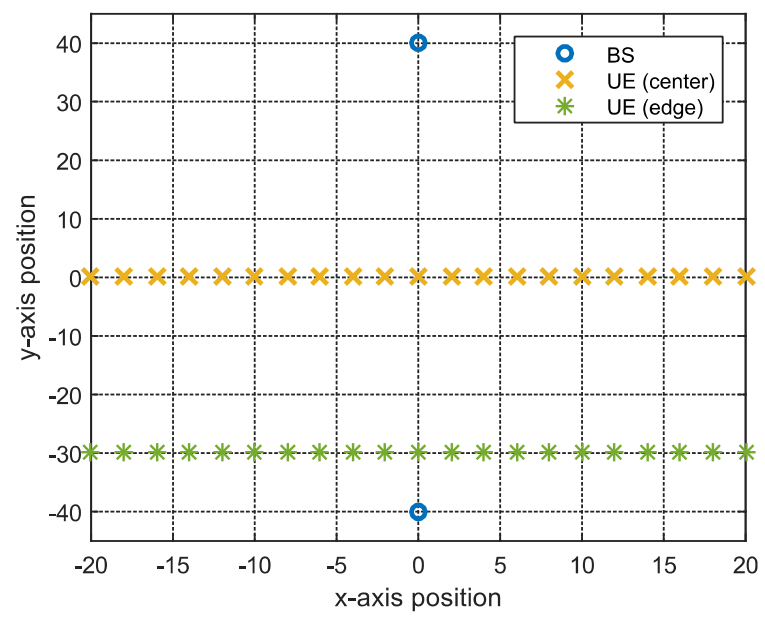

Fig. 3. The position of BSs and UEs. 


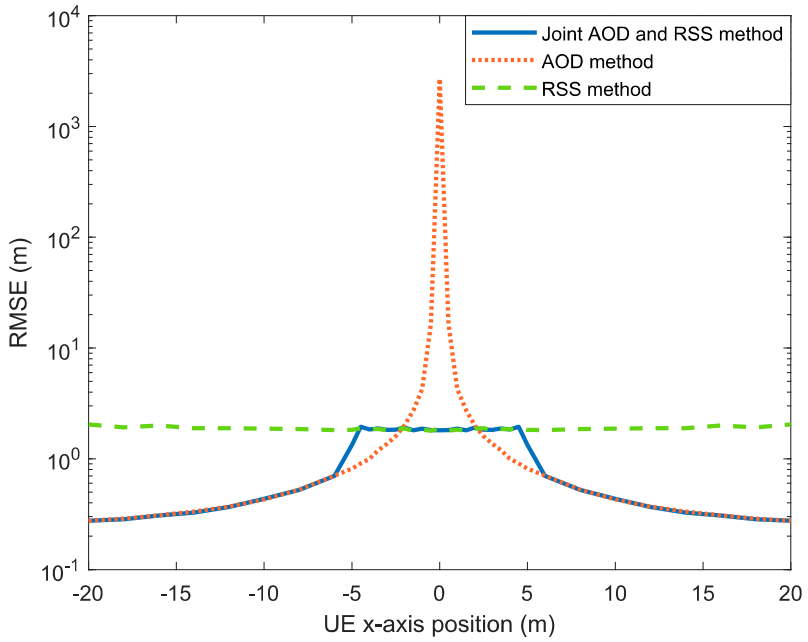

Fig. 4. RMSE of LE for various UE x-axis positions at the center of y-axis.

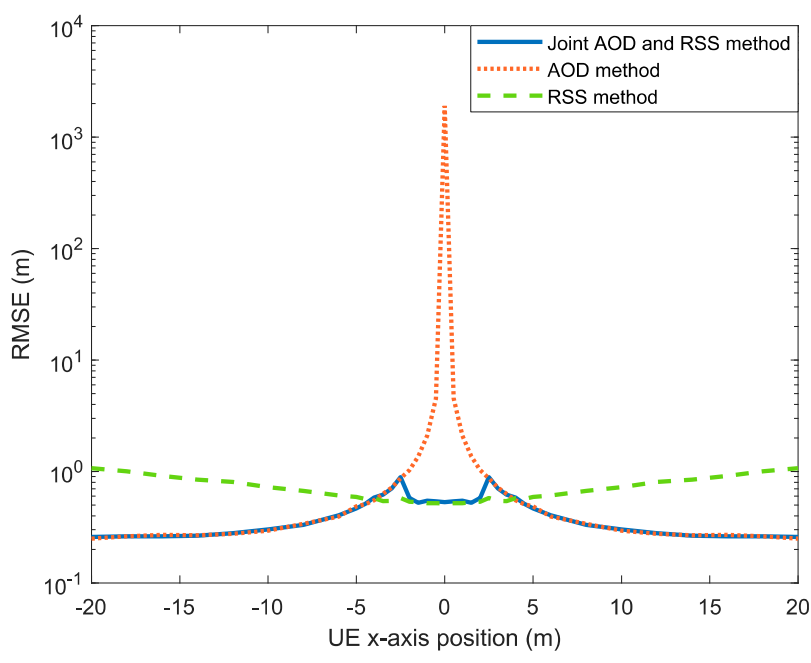

Fig. 5. RMSE of LE for various UE $x$-axis positions at the edge of $y$-axis.

In Fig. 4 and 5, the LE accuracy using AOD-only method is better than RSS-only method when the UE is not located directly between the two BS (around $<-5 \mathrm{~m}$ and $>5 \mathrm{~m}$ of the x-axis). However, the RSS-only method performs significantly better when the UE is located directly between the two BSs. This is as expected because, as we discussed in Sec. 3.3, a small angle error in AOD method will result to a high error when the UE is located on the direct line between the two BSs. By using the joint AOD and RSS method, AOD method can be switched to RSS method if the AOD method is calculated to be unreliable by using the Algorithm 1. The results in Fig. 4 and 5 confirm that the joint AOD and RSS method generally has lower RMSE than both AOD-only and RSS-only method across various UEs' locations. Note that the performance of RSS method is dependent on the distance between the UE and the BS. Larger BS-to-UE distance means lower signal to noise ratio (SNR) and higher RMSE. Therefore, RMSE in Fig. 4 must be higher than that of Fig. 5 for the RSS method. In addition, RMSE values for RSS method in Fig. 4 is more constant than that of Fig. 5. This is because, when you vary the UE's position across the $\mathrm{x}$-axis (refer to
Fig. 3), the UE at the center of y-axis will have less BS-toUE distance variation than the UE at the edge of $y$-axis.

Next, we observe the statistical distribution of LE error for AOD and RSS methods. Specifically, we observe their cumulative probability distributions at two locations which are $(x, y)=(20,0)$ (far from the direct line between the two BSs) and $(x, y)=(1,0)$ (near the direct line between the two BSs). The results are given in Fig. 6.

Figure 6 shows that the AOD method is more likely to have lower error than RSS method when the UE is located farther from the direct line between the two BSs (e.g. $(x, y)=(20,0))$. However, RSS method is more likely to have lower error than AOD method when the UE is located near the direct line between the two BSs (e.g. $(x, y)=(1,0))$. These results have confirmed the previous RMSE results which show that the AOD method performs better when UEs is not located directly between the two BSs while the RSS method performs better when the UE is located directly between the two BS. Using the proposed algorithm, the joint AOD and RSS method will have the same performance as AOD method when $(x, y)=(20,0)$ and the same performance as RSS method when $(x, y)=(1,0)$.

For the next test, we calculate the PAPR of OFDM signals that are generated by every antenna at the BS. The PAPR is calculated by dividing the peak power with the average power of the OFDM signal in time domain. The results are given in Fig. 7.

Figure 7 shows that the proposed random subcarrierbeamforming angle allocation method has average PAPR around $5 \mathrm{~dB}$, which is significantly less than that of linear allocation method, which has $16 \mathrm{~dB}$ PAPR. Therefore, the proposed beamforming signal has improved the practicality of the LE method by reducing the PAPR of the transmitted signal.

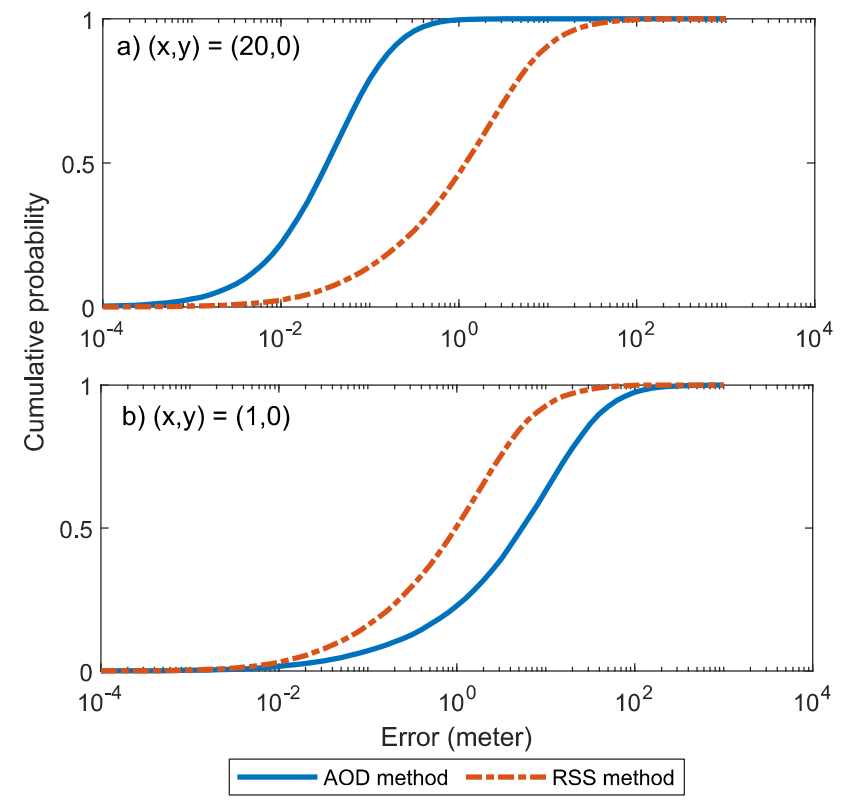

Fig. 6. Cumulative probability distributions of location estimation error for AOD and RSS methods at a) $(x, y)=(20,0)$ and $b)(x, y)=(1,0)$ of UE locations. 


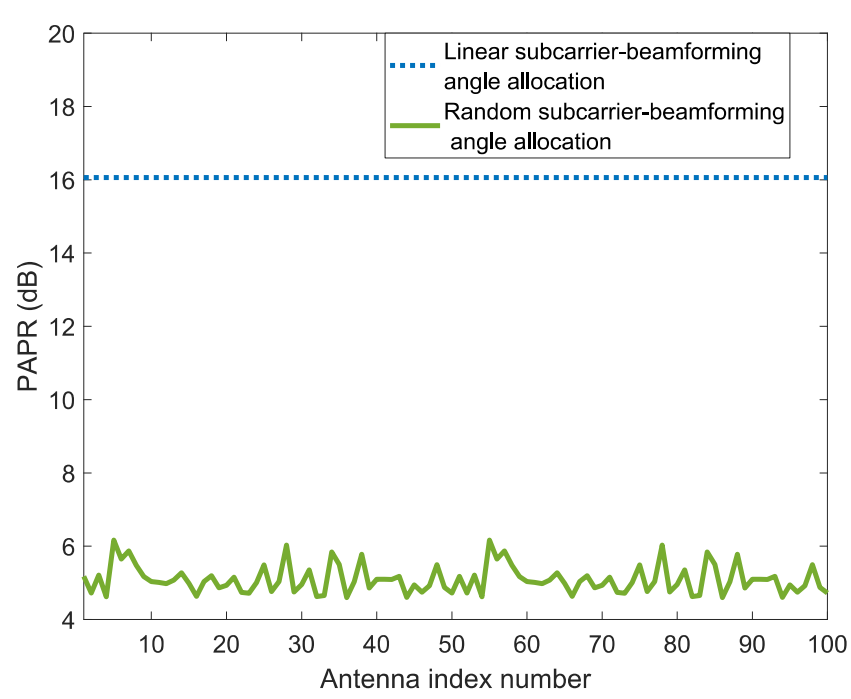

Fig. 7. PAPR of different beamforming allocation methods for various antenna index numbers.

\section{Conclusions}

In this paper, UE's location estimation method that uses joint AOD from BS and RSS at UE has been proposed. To achieve this, a special MIMO-OFDM beamforming signal has been designed to enable LE at each UE. In the proposed method, the number of UEs in the area does not affect the performance and calculation complexity of the LE because the LE is done independently at each UE. This paper also proposed to use random subcarrierbeamforming angle allocation method, which aims to reduce the PAPR of the transmitted signal and to improve the practicality of the proposed LE. The RMSE results across various UEs' locations show that the proposed method generally has higher estimation accuracy than that of AODonly and RSS-only LE methods. The PAPR of the beamforming signal has also been significantly reduced by using the proposed random subcarrier-beamforming allocation method.

\section{Acknowledgments}

This work is funded by the Faculty Program Research Grant, GPF029A-2018, from University of Malaya Faculty of Engineering.

\section{References}

[1] HOFMANN-WELLENHOF, B., LICHTENEGGER, H., COLLINS, J. Global Positioning System: Theory and Practice. New York (NY, USA): Springer, 2001. ISBN 978-3-7091-6199-9

[2] LIU, H., DARABI, H., BANERJEE, P., et al. Survey of wireless indoor positioning techniques and systems. IEEE Transactions on Systems, Man, and Cybernetics, Part $C$ (Applications and Reviews), 2007, vol. 37, no. 6, p. 1067-1080. DOI: 10.1109/TSMCC.2007.905750
[3] DARDARI, D., CLOSAS, P., DJURIĆ, P. M. Indoor tracking: Theory, methods, and technologies. IEEE Transactions on Vehicular Technology, 2015, vol. 64, no. 4, p. 1263-1278. DOI: 10.1109/TVT.2015.2403868

[4] GARCIA, N., WYMEERSCH, H., LARSSON, E. G., et al. Direct localization for massive MIMO. IEEE Transactions on Signal Processing, 2017, vol. 65, no. 10, p. 2475-2487. DOI: 10.1109/TSP.2017.2666779

[5] GUERRA, A., GUIDI, F., DARDARI, D. Single-anchor localization and orientation performance limits using massive arrays: MIMO vs. beamforming. IEEE Transactions on Wireless Communications, 2018, vol. 17 , no. 8, p. 5241-5255. DOI: 10.1109/TWC.2018.2840136

[6] SHAHMAnSOORI, A., GARCIA, G. E., DESTINO, G., et al. Position and orientation estimation through millimeter-wave MIMO in 5G systems. IEEE Transactions on Wireless Communications, 2018, vol. 17, no. 3, p. 1822-1835. DOI: 10.1109/TWC.2017.2785788

[7] SAVIC, V., LARSSON, E. G. Fingerprinting-based positioning in distributed massive MIMO systems. In 2015 IEEE 82nd Vehicular Technology Conference (VTC2015-Fall). Boston (MA, USA), 2015, p. 1-5. DOI: 10.1109/VTCFall.2015.7390953

[8] VIEIRA, J., LEITINGER, E., SARAJLIC, M., et al. Deep convolutional neural networks for massive MIMO fingerprintbased positioning. In Proceedings of the 28th IEEE Annual International Symposium on Personal, Indoor and Mobile Radio Communications. Montreal (QB, Canada), 2017, p. 1-6. DOI: 10.1109/PIMRC.2017.8292280

[9] HWANG, I., SONG, B., SOLIMAN, S. S. A holistic view on hyper-dense heterogeneous and small cell networks. IEEE Communications Magazine, 2013, vol. 51, no. 6, p. 20-27. DOI: 10.1109/MCOM.2013.6525591

[10] HEATH JR., R. W., WU, T., KWON, Y. H., et al. Multiuser MIMO in distributed antenna systems with out-of-cell interference. IEEE Transactions on Signal Processing, 2011, vol. 59, no. 10, p. 4885-4899. DOI: 10.1109/TSP.2011.2161985

[11] KRAMER, G., GASTPAR, M., GUPTA, P. Cooperative strategies and capacity theorems for relay networks. IEEE Transactions on Information Theory, 2005, vol. 51, no. 9, p. 3037-3063. DOI: 10.1109 /TIT.2005.853304

[12] CASTRO-ARVIZU, J. M., CLOSAS, P., FERNÁNDEZ-RUBIO, J. A. Cramér-Rao lower bound for breakpoint distance estimation in a path-loss model. In 2014 IEEE International Conference on Communications Workshops (ICC). Sydney (NSW, Australia), 2014, p. 176-180. DOI: 10.1109/ICCW.2014.6881192

[13] MORAGREGA, A., ClOSAS, P., IBARS, C. Potential game for energy-efficient RSS-based positioning in wireless sensor networks. IEEE Journal on Selected Areas in Communications, 2015 , vol. 33, no. 7, p. 1394-1406. DOI: 10.1109/JSAC.2015.2430172

[14] RUSEK, F., PERSSON, D., LAU, B., et al. Scaling up MIMO: Opportunities and challenges with very large arrays. IEEE Signal Processing Magazine, 2013, vol. 30, no. 1, p. 40-60. DOI: 10.1109/MSP.2011.2178495

[15] MOLISCH, A. F. Wireless Communications. 2nd ed. West Sussex (UK): JohnWiley \& Sons Ltd., 2011. ISBN: 978-0-470-74187-0

[16] SPENCER, Q. H., JEFFS, B. D., JENSEN, M. A., et al. Modeling the statistical time and angle of arrival characteristics of an indoor multipath channel. IEEE Journal on Selected Areas in Communications, 2000, vol. 18, no. 3, p. 347-360. DOI: $10.1109 / 49.840194$

[17] ITU-R. Propagation Data and Prediction Methods for the Planning of Indoor Radio Communication Systems and the Radio Local Area Networks in the Frequency Range $900 \mathrm{MHz}$ to 100 $G H z$. Recommendation ITU-R P.1238-8, 2015. Available at: https://www.itu.int/rec/R-REC-P.1238 
[18] NGO, H. Q., ASHIKHMIN, A., YANG, H., et al. Cell-free massive MIMO versus small cells. IEEE Transactions on Wireless Communications, 2017, vol. 16, no. 3, p. 1834-1850. DOI: 10.1109/TWC.2017.2655515

\section{About the Authors}

Wan Amirul MAHYIDDIN (corresponding author) received the M.Eng. degree from the Imperial College London in 2009, the M.Sc. degree from University of Malaya in 2012, and the Ph.D. degree from University of Canterbury in 2016 . He is currently a senior lecturer at the Department of Electrical Engineering, University of Malaya. His research interests are multiple antennas system, cooperative MIMO, channel modelling and positioning system.

Ahmad Loqman AHMAD MAZUKI received the M.Sc. degree in Engineering from UPNM, Malaysia in 2013. He is currently a lecturer at the Department of Electrical and Electronic Engineering, National Defense University of Malaysia. He is currently doing his Ph.D. at the Department of Electrical Engineering, Faculty of Engineering, University of Malaya. His research interests are sensors, robotic and passive radars.

Kaharudin DIMYATI graduated with B.Eng. from University of Malaya, Malaysia in 1992. He was awarded with
Ph.D. degree from the University of Wales Swansea, U.K., in 1996. He is currently a Professor at the Department of Electrical Engineering, Faculty of Engineering, University of Malaya. Since joining the university, he is actively involved in teaching, postgraduate supervision, research and also administration. He is a member of IEEE, IET and IEICE. He is a professional engineer and a Chartered Engineer.

Mohamadariff OTHMAN received the B.Eng. degree from the Malaysia Multimedia University, the M.Sc. degree in Engineering from Universiti Sains Malaysia, and the Ph.D. degree from Universiti Sains Malaysia. He is currently a senior lecturer in the Department of Electrical Engineering, University of Malaya. His research interest is antenna design.

Norrima MOKHTAR received the B.Eng. degree from University of Malaya, the M.Eng. and the Ph.D. degree from Oita Univerity, Japan. She is currently a senior lecturer in the Department of Electrical Engineering, University of Malaya. Her research interests are signal processing and human-machine interface.

Hamzah AROF obtained his Ph.D. from the University of Wales and is now affiliated with the Department of Electrical Engineering, University of Malaya. His current research interests include signal processing, photonics and econometrics. 